\title{
Measuring the dispersion of rainfall using Bayesian confidence intervals for coefficient of variation of delta- lognormal distribution: a study from Thailand
}

\author{
Noppadon Yosboonruang ${ }^{1}$, Sa-aat Niwitpong ${ }^{\text {Corresp., } 1}{ }^{1}$, Suparat Niwitpong ${ }^{1}$ \\ ${ }^{1}$ Department of Applied Statistics, Faculty of Applied Science, King Mongkut's University of Technology North Bangkok, Bangkok, Thailand \\ Corresponding Author: Sa-aat Niwitpong \\ Email address: sa-aat.n@sci.kmutnb.ac.th
}

Since rainfall data series often contain zero values and thus follow a delta-lognormal distribution, the coefficient of variation is often used to illustrate the dispersion of rainfall in a number of areas and so is an important tool in statistical inference for a rainfall data series. Therefore, the aim in this paper is to establish new confidence intervals for a single coefficient of variation for delta-lognormal distributions using Bayesian methods based on the independent Jeffreys', the Jeffreys' Rule, and the uniform priors compared with the fiducial generalized confidence interval. The Bayesian methods are constructed with either equitailed confidence intervals or the highest posterior density interval. The performance of the proposed confidence intervals was evaluated using coverage probabilities and expected lengths via Monte Carlo simulations. The results indicate that the Bayesian equitailed confidence interval based on the independent Jeffreys' prior outperformed the other methods. Rainfall data recorded in national parks in July 2015 and in precipitation stations in August 2018 in Nan province, Thailand are used to illustrate the efficacy of the proposed methods using a real-life dataset. 


\section{Measuring the dispersion of rainfall using Bayesian 2 confidence intervals for coefficient of variation of delta- 3 lognormal distribution: a study from Thailand

Noppadon Yosboonruang ${ }^{1}$, Sa-aat Niwitpong ${ }^{2}$, Suparat Niwitpong ${ }^{3}$

1,2,3 Department of Applied Statistics, Faculty of Applied Science, King Mongkut's University of Technology North Bangkok, Bangkok, Thailand

\section{Corresponding Author:}

Sa-aat Niwitpong ${ }^{1}$

1518 Pracharat 1 Road,Wongsawang, Bangsue, Bangkok, 10800, Thailand

Email address: sa-aat.n@sci.kmutnb.ac.th

\section{Abstract}

Since rainfall data series often contain zero values and thus follow a delta-lognormal distribution, the coefficient of variation is often used to illustrate the dispersion of rainfall in a number of areas and so is an important tool in statistical inference for a rainfall data series. Therefore, the aim in this paper is to establish new confidence intervals for a single coefficient of variation for deltalognormal distributions using Bayesian methods based on the independent Jeffreys', the Jeffreys' Rule, and the uniform priors compared with the fiducial generalized confidence interval. The Bayesian methods are constructed with either equitailed confidence intervals or the highest posterior density interval. The performance of the proposed confidence intervals was evaluated using coverage probabilities and expected lengths via Monte Carlo simulations. The results indicate that the Bayesian equitailed confidence interval based on the independent Jeffreys' prior outperformed the other methods. Rainfall data recorded in national parks in July 2015 and in precipitation stations in August 2018 in Nan province, Thailand are used to illustrate the efficacy of the proposed methods using a real-life dataset.

\section{Introduction}

Presently, the effects of global climate change caused by many factors, both natural and man-made (such as fuel burning, burning forests, deforestation, and oil drilling), are continuous. Such factors directly enhance natural changes such as the greenhouse effect and cause changes in precipitation, sea level, and the polar vortex. Thailand is a country that has been affected, as has been seen in the past few years. Especially in the north of Thailand, a lot of deforestation has caused flooding because there are insufficient trees to absorb water due to heavy rain. Subsequently, many organizations, both governmental and from the private sector, are interested in finding ways to mitigate the damage from such events, and thus a study on measuring the dispersion of rainfall in 
40

41

42

43

44

45

46

47

48

49

50

51

52

53

54

55

56

57

58

59

60

61

62

63

64

65

66

67

68

69

70

71

72

73

74

75

76

77

78

areas with the potential risk of flooding has become necessary. In statistics, the measurement of the coefficient of variation of rainfall data can illustrate the dispersion of rainfall and also predict the precipitation in each area as well. However, rainfall data are often zero-inflated, especially during the winter to summer months (October to May in Thailand; Climatological group (2015)). Thus, the rainfall dataset follows a combination of two distributions: lognormal and binomial. Therefore, rainfall data follows a delta-lognormal distribution, as has been reported by many researchers (e.g. Fukushi (1988); Shimizu (1993); Yue (2000); Kong et al. (2012)).

In many scientific studies, the data consist of positive right-skewed observations with an excess of exact zeros, and Aitchison (1955) determined that the distribution of this data is deltalognormal, which has subsequently been used in other studies: for instance, the diagnostic charge data in Callahan's (Callahan et al.,1997) study (Zhou and Tu, 2000; Li et al., 2013), fisheries data from a trawler survey carried out by the National Institute of Water and Atmospheric Research in New Zealand (Fletcher, 2008; Wu and Hsieh, 2014), household expenditure explored by the Ministry of Food in 1950 (Aitchison, 1955), and the concentration of airborne chlorine measured at an industrial site in the US (Owen and DeRouen, 1980; Tian and Wu, 2006).

To solve the problems when dealing with delta-lognormally distributed data, statistical inference is used which constructs confidence intervals for the parameters of interest, and in the past two decades, many researchers have investigated this. Kvanli et al. (1998) constructed a confidence interval based on the likelihood ratio test approach for the population mean when there are many zeros in the data. Zhou and Tu (2000) proposed three different interval estimation procedures comprising a percentile- $t$ bootstrap interval based on sufficient statistics and two likelihood-based confidence intervals for the mean of diagnostic test charge data containing zeros. Chen and Zhou (2006) introduced confidence intervals based on a true generalized pivotal (GP) method, an approximate GP method, a signed log-likelihood ratio (SLLR), and a modified SLLR for the ratio or difference between two means of lognormal populations with zeros. Tian and Wu (2006) constructed confidence intervals for the mean of lognormal data with excess zeros using an adjusted SLLR via an SLLR approach and a bootstrap approach. Fletcher (2008) used three methods, namely Aitchison's estimator, a modification of Cox's method for lognormal distributions, and a profile-likelihood interval to construct confidence intervals for the mean of a delta-lognormal distribution. Buntao and Niwitpong (2013) presented two confidence intervals: the concept of the GP approach (GPA) and the method of variance estimate recovery (MOVER) for the ratio of coefficients of variation of delta-lognormal distributions. Li et al. (2013) proposed two methods for the mean based on an approximate GP quantity and fiducial quantity of lognormal data with excess zeros. Wu and Hsieh (2014) established confidence intervals with Aitchison's method, a modified Land's method, the profile-likelihood interval, and the generalized confidence interval (GCI) for the mean of a delta-lognormal distribution. Maneerat et al. (2018) introduced GCI, MOVER based on the variance stabilizing transformation (VST), the Wilson score interval, and Jeffreys' method to construct confidence intervals for the mean of a delta-lognormal distribution.

PeerJ reviewing PDF | (2019:03:35675:2:0:NEW 17 Jun 2019) 
The coefficient of variation is another interesting parameter defined as the ratio of the standard deviation to the mean. It is useful to describe the dispersion of data and can be used to compare the degree of variation between two or more datasets with different measurement units. The coefficient of variation is used in several fields, such as medical science, meteorology, agriculture and economics (e.g., Kim et al. (2005); Gulhar et al. (2012); Tian (2005)). Recently, several researchers have considered various approaches to construct confidence intervals for coefficients of variation. For example, Wong and Wu (2002), Tian (2005), Mahmoudvand and Hassani (2009), Donner and Zou (2012), and Wongkhao et al. (2015) established confidence intervals for the coefficient of variation for a normal distribution. After that, van Zyl and van der Merwe (2017) proposed a Bayesian control chart for the common coefficient of variation for a normal distribution. In studies on two-parameter exponential distributions, Sangnawakij and Niwitpong (2017) used three methods, namely MOVER, GCI, and the asymptotic confidence interval, to establish confidence intervals for a single coefficient of variation and the difference between coefficients of variation, and Thangjai and Niwitpong (2017) presented confidence intervals based on an adjusted MOVER, GCI, and a large sample method for weighted coefficients of variation.

There have been studies on other skewed distributions, such as the one by Fletcher (2008) who presented three methods: Aitchison's estimator (the classical method), a modification of Cox's method for the lognormal, and a profile-likelihood interval, to construct confidence intervals for the mean of a delta-lognormal distribution. Fletcher suggested that Cox's method and profile likelihood interval, which are the modified methods, are well performed to construct the confidence intervals for the mean of a delta-lognormal distribution. While Aitchison's estimator tend to have too low an upper limit. Therefore, Fletcher not recommend the Aitchison's estimator. Buntao and Niwitpong (2012) revealed the GPA and a closed-form method of variance estimation for coefficients of variation for both lognormal and delta-lognormal distributions. Harvey and van der Merwe (2012) constructed confidence intervals for means and variances of lognormal and bivariate lognormal distributions using a Bayesian method. Niwitpong (2013) presented a new confidence interval for the coefficient of variation of a lognormal distribution with restricted parameters. D'Cunha and Rao (2014) offered a Bayes confidence interval for the mean of a lognormal distribution and compared it with the maximum likelihood estimator method. Sangnawakij et al. (2015) proposed MOVER with Score and Wald interval methods to construct confidence intervals for the ratio of coefficients of variation of gamma distributions. Rao and D'Cunha (2016) presented Bayesian confidence intervals for the median of a lognormal distribution and compared it with the confidence interval obtained from a Monte Carlo simulation. Recently, Yosboonruang et al. (2018) constructed confidence intervals for the coefficient of variation of a delta-lognormal distribution based on a modified Fletcher method using the concept of Fletcher (2008), and the GCI. The modified Fletcher, based on its variance, is the basic method to construct the confidence interval. Although this method failed in term of the coverage probability and the expected length, it is used to compare with the GCI. Moreover, they proposed methods including the fiducial generalized confidence interval (FGCI) and MOVER based on the 
119 VST, the Wilson score, and Jeffreys' method to establish the confidence intervals for the 120 coefficient of variation of three parameters of a delta-lognormal distribution, of which FGCI is recommended for constructing confidence intervals (Yosboonruang et al., 2019). In addition, they extended this study to construct confidence intervals for the coefficient of variation.

The goal of this study is to propose new confidence intervals using Bayesian methods and comparing them with FGCI proposed by Yosboonruang et al. (2019) for a single coefficient of variation of a delta-lognormal distribution. The methods and theories to establish the confidence intervals are described in Section 2. Next, a simulation study and results are presented in Section 3 , and then the proposed methods are applied to the real-world datasets, as detailed in Section 4. The last section contains conclusions on the study.

\section{Methods}

Let $\boldsymbol{V}=\left(V_{1}, V_{2}, \ldots, V_{n}\right)$ be a positive random variable from a lognormal distribution with

$$
f\left(v_{i} ; \mu, \sigma^{2}\right)= \begin{cases}\frac{1}{v_{i} \sigma \sqrt{2 \pi}} \exp \left\{-\frac{1}{2 \sigma^{2}}\left[\ln \left(v_{i}\right)-\mu\right]^{2}\right\} & ; \quad v_{i}>0 \\ 0 \quad & ; \text { otherwise. }\end{cases}
$$

Suppose that the population of interest contains both zero and non-zero observed values, denoted by $n_{(0)}$ and $n_{(1)}$, respectively, where $n=n_{(0)}+n_{(1)}$. The zero observations follow a binomial distribution, $n_{(0)} \sim \operatorname{Bin}\left(n, \delta^{\prime}\right)$, where $\delta^{\prime}=1-\delta$ is the probability of zero observations, and the non-zero observations follow a lognormal distribution, thus resulting in a delta-lognormal distribution. Let $\boldsymbol{X}=\left(X_{1}, X_{2}, \ldots, X_{n}\right)$ be a random sample from a delta-lognormal distribution, denoted by $\Delta\left(\delta^{\prime}, \mu, \sigma^{2}\right)$. The distribution function of a delta-lognormal population presented by Tian and $\mathrm{Wu}(2006)$ can be derived as

$$
G\left(x_{i} ; \delta^{\prime}, \mu, \sigma^{2}\right)= \begin{cases}\delta^{\prime} & ; x=0 \\ \delta^{\prime}+\delta F\left(x_{i} ; \mu, \sigma^{2}\right) & ; x>0\end{cases}
$$

144 where $F\left(x_{i} ; \mu, \sigma^{2}\right)$ is the lognormal cumulative distribution function. Let $145 Y_{i}=\ln \left(X_{i}\right) \sim N\left(\mu, \sigma^{2}\right)$ for $X_{i}>0$. Aitchison (1955) described the respective population mean 146 and variance of $X$ as 


$$
E(X)=\mu_{X}=\delta \exp \left(\mu+\frac{\sigma^{2}}{2}\right)
$$

148 and

149

150

$$
\operatorname{Var}(X)=\sigma_{X}^{2}=\delta \exp \left(2 \mu+\sigma^{2}\right)\left[\exp \left(\sigma^{2}\right)-\delta\right]
$$

$$
\hat{\mu}_{X}=\hat{\delta} \exp (\hat{\mu}) \psi_{n_{(1)}}\left(\frac{\hat{\sigma}^{2}}{2}\right)
$$

154

155 where $\hat{\delta}=\frac{n_{(1)}}{n}, \hat{\mu}=\frac{1}{n_{(1)}} \sum_{i=1}^{n_{(1)}} \ln \left(x_{i}\right)$, and $\hat{\sigma}^{2}=\frac{1}{n_{(1)}-1} \sum_{i=1}^{n_{(1)}}\left[\ln \left(x_{i}\right)-\hat{\mu}\right]^{2}$, then the coefficient of 156 variation of $X$ can be expressed as

157

$$
C V(X)=\eta=\sqrt{\frac{\exp \left(\sigma^{2}\right)}{\delta}-1}
$$

158 159 160 161 162 163 165 168 observation is written as section. likelihood function is given by

$$
L\left(\delta^{\prime}, \mu, \sigma^{2} \mid \boldsymbol{x}\right) \propto\left(\delta^{\prime}\right)^{n_{(0)}} \delta^{n_{(1)}} \prod_{i=1}^{n_{(1)}} \frac{1}{\sqrt{\sigma^{2}}} \exp \left\{-\frac{1}{2 \sigma^{2}}\left[\ln \left(x_{i}\right)-\mu\right]^{2}\right\} .
$$

\section{The Bayesian confidence interval for a single coefficient of variation}

If a delta-lognormal distribution has three unknown parameters $\left(\delta^{\prime}, \mu, \sigma^{2}\right)$, then the joint

Therefore, the Fisher Information matrix of the unknown parameters $\left(\delta^{\prime}, \mu, \sigma^{2}\right)$ per unit 


$$
I\left(\delta^{\prime}, \mu, \sigma^{2}\right)=\left[\begin{array}{ccc}
\frac{n}{\delta^{\prime} \delta} & 0 & 0 \\
0 & \frac{n \delta}{\sigma^{2}} & 0 \\
0 & 0 & \frac{n \delta}{2\left(\sigma^{2}\right)^{2}}
\end{array}\right]
$$

169

170

171

172

173

174

175

176

177

178

179

180

181

182

183

$$
\begin{aligned}
p\left(\delta^{\prime}\right) & \propto \sqrt{\left|I\left(\delta^{\prime}\right)\right|} \\
& \propto\left(\delta^{\prime}\right)^{-\frac{1}{2}} \delta^{-\frac{1}{2}},
\end{aligned}
$$

184

185

186

187

188

189

190

191

192

In the following section, the Bayesian confidence interval is constructed upon three priors: the independent Jeffreys, Jeffreys' Rule, and uniform.

\section{The Bayesian confidence interval using the independent Jeffreys' prior}

Jeffreys' prior is defined as $p(\theta) \propto \sqrt{|I(\theta)|}$, where $I(\theta)$ is a Fisher Information matrix. It is a non-informative prior distribution used in Bayesian parameter estimation and is very useful because it has the notable property of invariance under the reparameterization of $\theta$ (Harold Jeffreys, 1946).

Independent Jeffreys' prior is a non-informative prior under the concept of establishing the product of Jeffreys' prior for each parameter while imposing staticity on the others (Rubio and Liseo, 2014).

For a binomial distribution, the parameter of interest is the probability $\delta^{\prime}$, then the Jeffreys' invariant prior for a binomial parameter is given by

which is $\operatorname{Beta}(1 / 2,1 / 2)$ (Bolstad and Curran, 2017). Subsequently, the posterior distribution of $\delta^{\prime}$ is in the form

$$
p\left(\delta^{\prime} \mid n_{(0)}\right) \propto\left(\delta^{\prime}\right)^{n_{(0)}-\frac{1}{2}} \delta^{n_{(1)}-\frac{1}{2}}
$$

which is a beta distribution $\operatorname{Beta}\left(n_{(0)}+1 / 2, n_{(1)}+1 / 2\right)$. Similarly, the independent Jeffreys' prior for a lognormal distribution is $p\left(\sigma^{2}\right) \propto \sigma^{-2}$. Therefore, the prior distribution for a delta-lognormal distribution can be expressed as

$$
p\left(\delta^{\prime}, \sigma^{2}\right) \propto \sigma^{-2}\left(\delta^{\prime}\right)^{-\frac{1}{2}} \delta^{-\frac{1}{2}}
$$


193 The joint posterior density function is clearly defined as

194

$$
\begin{aligned}
p\left(\delta^{\prime}, \sigma^{2} \mid \boldsymbol{x}\right)= & \frac{1}{\operatorname{Beta}\left(n_{(0)}+\frac{1}{2}, n_{(1)}+\frac{1}{2}\right)}\left(\delta^{\prime}\right)^{n_{(0)}-\frac{1}{2}} \delta^{n_{(1)}-\frac{1}{2}} \\
& \times \frac{1}{\sqrt{2 \pi} \frac{\sigma}{\sqrt{n_{(1)}}}} \exp \left[-\frac{1}{2 \frac{\sigma^{2}}{n_{(1)}}}(\mu-\hat{\mu})^{2}\right] \\
& \times \frac{\left[\frac{\left(n_{(1)}-1\right) \hat{\sigma}^{2}}{2}\right]^{\frac{n_{(1)}-1}{2}}}{\Gamma\left(\frac{n_{(1)}-1}{2}\right)}\left(\sigma^{2}\right)^{-1-\frac{\left(n_{(1)}-1\right)}{2}} \exp \left[-\frac{\left(n_{(1)}-1\right) \hat{\sigma}^{2}}{2 \sigma^{2}}\right],
\end{aligned}
$$

195

196 where $\hat{\mu}=\frac{1}{n_{(1)}} \sum_{i=1}^{n_{(1)}} \ln \left(x_{i}\right)$ and $\hat{\sigma}^{2}=\frac{1}{n_{(1)}-1} \sum_{i=1}^{n_{(1)}}\left[\ln \left(x_{i}\right)-\hat{\mu}^{2}\right]^{2}$. Since $\delta^{\prime}$ and $\sigma^{2}$ are

197 independent, then the posterior distributions of $\delta^{\prime}$ and $\sigma^{2}$ are a beta and an inverse gamma 198 distribution, respectively, as follows:

199

$$
\delta^{\prime} \mid \boldsymbol{x} \sim \operatorname{Beta}\left(n_{(0)}+\frac{1}{2}, n_{(1)}+\frac{1}{2}\right)
$$

201 and

202

$$
p\left(\sigma^{2} \mid \boldsymbol{x}\right)=\frac{\left[\frac{\left(n_{(1)}-1\right) \hat{\sigma}^{2}}{2}\right]^{\frac{n_{(1)}-1}{2}}}{\Gamma\left(\frac{n_{(1)}-1}{2}\right)}\left(\sigma^{2}\right)^{-1-\frac{\left(n_{(1)}-1\right)}{2}} \exp \left[-\frac{\left(n_{(1)}-1\right) \hat{\sigma}^{2}}{2 \sigma^{2}}\right] .
$$

204 To construct the Bayesian confidence interval, $\delta$ and $\sigma^{2}$ in Equation (6) are substituted by $\delta^{\prime} \mid \boldsymbol{x}$ 205 and $\sigma^{2} \mid \boldsymbol{x}$ defined in Equations (13) and (14), respectively. Therefore, the $100(1-\alpha) \%$ two206 sided confidence interval for the coefficient of variation based on the independent Jeffreys' prior 207 Bayesian is obtained by 


$$
C I_{\eta}^{B . i n d j}=\left[L_{\eta}^{B . i n d j}, U_{\eta}^{B . i n d j}\right]
$$

where $L_{\eta}^{\text {B.indj }}$ and $U_{\eta}^{\text {B.indj }}$ are the lower and upper bounds of the $100(1-\alpha) \%$ equitailed confidence interval and the highest posterior density (HPD) interval of $\eta$, respectively.

The HPD interval is an interval in the domain of a posterior probability distribution which gives the narrowest length of the interval (Hyndman, 1995; Yau and Campbell, 2019). It represents the most credible points which cover most of the distribution. In addition, each point inside the interval has a higher probability density than those outside it.

\section{The Bayesian confidence interval using the Jeffreys' Rule prior}

As mentioned previously, Jeffreys' Rule prior is obtained from the square root of the determinant of the Fisher Information matrix. This prior is appropriate for a single parameter. The Jeffreys' Rule prior has the rule that the prior is invariant (the valuable property) (Lee, 2012), which is imposed as $p\left(\sigma^{2}\right) \propto \sigma^{-3}$. From Harvey and van der Merwe (2012), the Jeffreys' Rule prior for $\delta^{\prime}$

221 in a binomial distribution is $p\left(\delta^{\prime}\right) \propto\left(\delta^{\prime}\right)^{-\frac{1}{2}} \delta^{\frac{1}{2}}$. It is easy to find the Jeffreys' Rule prior for the 222 delta-lognormal distribution, which is defined as

$$
p\left(\delta^{\prime}, \sigma^{2}\right) \propto \sigma^{-3}\left(\delta^{\prime}\right)^{-\frac{1}{2}} \delta^{\frac{1}{2}}
$$

$$
\begin{aligned}
p\left(\delta^{\prime}, \sigma^{2} \mid \boldsymbol{x}\right)= & \frac{1}{\operatorname{Beta}\left(n_{(0)}+\frac{1}{2}, n_{(1)}+\frac{3}{2}\right)}\left(\delta^{\prime}\right)^{n_{(0)}-\frac{1}{2}} \delta^{n_{(1)}+\frac{1}{2}} \\
& \times \frac{1}{\sqrt{2 \pi} \frac{\sigma}{\sqrt{n_{(1)}}}} \exp \left[-\frac{1}{2 \frac{\sigma^{2}}{n_{(1)}}}(\mu-\hat{\mu})^{2}\right] \\
& \times \frac{\left[\frac{n_{(1)} \hat{\sigma}^{2}}{2}\right]^{\frac{n_{(1)}}{2}}}{\Gamma\left(\frac{n_{(1)}}{2}\right)}\left(\sigma^{2}\right)^{-1-\frac{n_{(1)}}{2}} \exp \left[-\frac{n_{(1)} \hat{\sigma}^{2}}{2 \sigma^{2}}\right],
\end{aligned}
$$


227 where $\hat{\mu}=\frac{1}{n_{(1)}} \sum_{i=1}^{n_{(1)}} \ln \left(x_{i}\right)$ and $\hat{\sigma}^{2}=\frac{1}{n_{(1)}-1} \sum_{i=1}^{n_{(1)}}\left[\ln \left(x_{i}\right)-\hat{\mu}\right]^{2}$. In addition, the posterior density

228 of $\delta^{\prime}$ becomes

229

230

$$
\delta^{\prime} \mid \boldsymbol{x} \sim \operatorname{Beta}\left(n_{(0)}+\frac{1}{2}, n_{(1)}+\frac{3}{2}\right)
$$

231 and the posterior distribution of $\sigma^{2}$ can be expressed as

232

$$
p\left(\sigma^{2} \mid \boldsymbol{x}\right)=\frac{\left[\frac{n_{(1)} \hat{\sigma}^{2}}{2}\right]^{\frac{n_{(1)}}{2}}}{\Gamma\left(\frac{n_{(1)}}{2}\right)}\left(\sigma^{2}\right)^{-1-\frac{n_{(1)}}{2}} \exp \left[-\frac{n_{(1)} \hat{\sigma}^{2}}{2 \sigma^{2}}\right] .
$$

233

234 Next, the confidence limit of $\eta$ is constructed using $\delta^{\prime} \mid \boldsymbol{x}$ and $\sigma^{2} \mid \boldsymbol{x}$ given by Equations (18) and 235 (19), respectively. Therefore, the $100(1-\alpha) \%$ equitailed confidence interval and HPD interval 236 for the coefficient of variation based on the Jeffreys' Rule prior Bayesian are obtained by

$$
C I_{\eta}^{\text {B.jrule }}=\left[L_{\eta}^{\text {B.jrule }}, U_{\eta}^{B . j \text { rule }}\right]
$$

where $L_{\eta}^{\text {B.jrule }}$ and $U_{\eta}^{B . j r u l e}$ are the lower and upper bounds of the confidence limit, respectively.

\section{The Bayesian confidence interval using the uniform prior}

242 The prior probability of the uniform prior is a constant function (Stone, 2013). This means that the 243 uniform prior gives equally likely a priori to all possible values (O'Reilly and Mars, 2015). The 244 uniform prior for the binomial proportion is $p\left(\delta^{\prime}\right) \propto 1$ (Bolstad and Curran, 2017), that for $\sigma^{2}$ is $245 p\left(\sigma^{2}\right) \propto 1$ (Kalkur T. and Rao, 2017), and that of a delta-lognormal distribution is $p\left(\delta^{\prime}, \sigma^{2}\right) \propto 1$.

246 The joint posterior density function can be expressed as 


$$
\begin{aligned}
p\left(\delta^{\prime}, \sigma^{2} \mid \boldsymbol{x}\right)= & \frac{1}{\operatorname{Beta}\left(n_{(0)}+1, n_{(1)}+1\right)}\left(\delta^{\prime}\right)^{n_{(0)}} \delta^{n_{(1)}} \frac{1}{\sqrt{2 \pi} \frac{\sigma}{\sqrt{n_{(1)}}}} \exp \left[-\frac{1}{2 \frac{\sigma^{2}}{n_{(1)}}}(\mu-\hat{\mu})^{2}\right] \\
& \times \frac{\left[\frac{\left(n_{(1)}-2\right) \hat{\sigma}^{2}}{2}\right]^{\frac{n_{(1)}-2}{2}}}{\Gamma\left(\frac{n_{(1)}-2}{2}\right)}\left(\sigma^{2}\right)^{-1-\frac{\left(n_{(1)}-2\right)}{2}} \exp \left[-\frac{\left(n_{(1)}-2\right) \hat{\sigma}^{2}}{2 \sigma^{2}}\right],
\end{aligned}
$$

248 where $\hat{\mu}=\frac{1}{n_{(1)}} \sum_{i=1}^{n_{(1)}} \ln \left(x_{i}\right)$ and $\hat{\sigma}^{2}=\frac{1}{n_{(1)}-1} \sum_{i=1}^{n_{(1)}}\left[\ln \left(x_{i}\right)-\hat{\mu}\right]^{2}$. By Equation (21), the respective 249 posterior distributions of $\delta^{\prime}$ and $\sigma^{2}$ are formed as

250

$$
\delta^{\prime} \mid \boldsymbol{x} \sim \operatorname{Beta}\left(n_{(0)}+1, n_{(1)}+1\right)
$$

252 and

253

$$
p\left(\sigma^{2} \mid \boldsymbol{x}\right)=\frac{\left[\frac{\left(n_{(1)}-2\right) \hat{\sigma}^{2}}{2}\right]^{\frac{n_{(1)}-2}{2}}}{\Gamma\left(\frac{n_{(1)}-2}{2}\right)}\left(\sigma^{2}\right)^{-1-\frac{\left(n_{(1)}-2\right)}{2}} \exp \left[-\frac{\left(n_{(1)}-2\right) \hat{\sigma}^{2}}{2 \sigma^{2}}\right],
$$

255 which are beta and inverse gamma distributions, respectively. From Equations (22) and (23), the 256 confidence limit for $\eta$ can be established, and consequently, the $100(1-\alpha) \%$ equitailed 257 confidence interval and HPD interval for the coefficient of variation based on the uniform prior 258 Bayesian are as follows:

$$
C I_{\eta}^{\text {B.uni }}=\left[L_{\eta}^{\text {B.uni }}, U_{\eta}^{\text {B.uni }}\right]
$$

261 where $L_{\eta}^{\text {B.uni }}$ and $U_{\eta}^{\text {B.uni }}$ are the lower and upper bounds of the confidence limit, respectively.

\section{Algorithm 1}

264 Step 1: Generate $\boldsymbol{x}_{i}, i=1,2, \ldots, n$ from a delta-lognormal distribution. 
265 Step 2: Compute $\hat{\delta}$ and $\hat{\sigma}^{2}$.

266 Step 3: Generate $\delta^{\prime} \mid \boldsymbol{x}$, which is the beta distribution from Equations (13), (18), and (22).

267 Step 4: Generate $\sigma^{2} \mid \boldsymbol{x}$, which is the inverse gamma distribution from Equations (14), (19), and 268 (23).

269 Step 5: Compute $\eta$ by substituting $\delta^{\prime} \mid \boldsymbol{x}$ and $\sigma^{2} \mid \boldsymbol{x}$ in Equation (6).

270 Step 6: Repeat Steps 3-5 5,000 times and obtain an array of $\eta$.

271 Step 7: Compute the 95\% equitailed confidence interval and HPD interval for $\eta$ from Equations 272 (15), (20), and (24). If $L \leq \eta \leq U$, then set $c p=1$; else, set $c p=0$.

273 Step 8: Repeat Steps 1-7 15,000 times to compute the coverage probability and the expected length.

274

275

276

277

278

279

280

281

282

283

284

285

286

287

288

289

290

291

292

\section{The FGCI for a single coefficient of variation}

The fiducial approach was first introduced by Fisher (1930), after which it has been used to construct confidence limits by many researchers, such as Hannig et al. (2006a), Hannig et al. (2006b), Hannig (2009), Hannig and Lee (2009), Li et al. (2013), and Yosboonruang et al. (2019). The concept of FGCI uses the respective generalized fiducial quantities for $\delta$ and $\sigma^{2}$ (Li et al., 2013):

$$
R_{\delta} \sim \frac{1}{2} \operatorname{Beta}\left(n_{(1)}, n_{(0)}+1\right)+\frac{1}{2} \operatorname{Beta}\left(n_{(1)}+1, n_{(0)}\right)
$$

and

$$
R_{\sigma^{2}}=\frac{\left(n_{(1)}-1\right) \hat{\sigma}^{2}}{U}
$$

where $U \sim \chi_{n_{(1)-1}^{-1}}^{2}$. Subsequently, the generalized fiducial quantity for $\eta$ is

$$
R_{\eta}=\sqrt{\frac{\exp \left(R_{\sigma^{2}}\right)}{R_{\delta}}-1} .
$$

Therefore, the $100(1-\alpha) \%$ generalized fiducial quantity interval for the coefficient of variation is defined by

$$
C I_{\eta}^{f g c i}=\left[R_{\eta}(\alpha / 2), R_{\eta}(1-\alpha / 2)\right]
$$


where $R_{\eta}(\alpha / 2)$ and $R_{\eta}(1-\alpha / 2)$ are the $100(\alpha / 2)$-th and $100(1-\alpha / 2)$-th percentiles of the 294 distribution of $R_{\eta}$, respectively.

295

296

297

298

299

300

\section{Algorithm 2}

Step 1: Generate $\boldsymbol{x}_{i}, i=1,2, \ldots, n$ from a delta-lognormal distribution.

Step 2: Compute $\hat{\delta}$ and $\hat{\sigma}^{2}$.

Step 3: Generate $\operatorname{Beta}\left(n_{(1)}, n_{(0)}+1\right)$ and $\operatorname{Beta}\left(n_{(1)}+1, n_{(0)}\right)$.

Step 4: Compute $R_{\delta}, R_{\sigma^{2}}$, and $R_{\eta}$ from Equations (25), (26), and (27), respectively.

Step 5: Repeat Steps 3-4 5,000 times and obtain an array of $R_{\eta}$.

Step 6: Compute the 95\% confidence intervals for $\eta$ from Equation (28). If $L \leq \eta \leq U$, then set $c p=1$; else, set $c p=0$.

Step 7: Repeat Steps 1-6 15,000 times to compute the coverage probability and the expected length.

\section{Results}

To evaluate the performance of the proposed methods, their coverage probabilities and expected lengths were estimated via Monte Carlo simulation using the $\mathrm{R}$ statistical programming language (Venables and Smith, 2009). Normally, the best confidence intervals are chosen from the coverage probability that is greater than or closest to the nominal confidence level and has the shortest expected lengths. In the simulation study, sample size $n$ was set as 25, 50,100, and 200; $\mu$ as 0 ; $\delta$ as $0.2,0.5,0.8$, and 0.9 ; and $\sigma^{2}$ as $0.1,0.5,1.0$, and 2.0. We eliminated the case of $n=25$, $\delta=0.2$ and $\sigma^{2}=0.1,0.5,1.0$, and 2.0 because the expected non-zero observations were less than 10 (see Fletcher (2008); $\mathrm{Wu}$ and Hsieh (2014)). For all of the simulations, the number of replications was set as 15,000, and 5,000 repetitions were used for the Bayesian and FGCI methods; the nominal confidence level was 0.95 .

The results in Table 1 show that the Bayesian method using the independent Jeffreys' prior for the equitailed confidence interval outperformed the others because the coverage probabilities were consistently greater than or close to the target in all cases. In addition, for the equitailed confidence intervals, the coverage probabilities of the Bayesian using the Jeffreys' Rule prior were less than the nominal confidence level of 0.95 for some of the cases: $n=25, \delta=0.5, \sigma^{2}=0.1$, $2.0 ; n=50,100, \delta=0.2, \sigma^{2}=0.1,2.0$; and $n=200, \delta=0.2, \sigma^{2}=0.1$. For the Bayesian method using the uniform prior, the coverage probabilities were close to 1 in a few cases when the sample sizes were less than 100 and had small variances together with high proportion of non-zero values. For the method with HPD intervals, the coverage probabilities of the independent Jeffreys' prior did not cover the target in most cases, especially for large sample sizes. Similarly, a few cases with the Bayesian method using the uniform prior had coverage probabilities less than the nominal confidence level when the sample sizes were large. Moreover, the Bayesian method using the Jeffreys' Rule prior had coverage probabilities of less than 0.95 in almost all cases. Last, the 
330 coverage probabilities with FGCI did not cover the nominal confidence level when the variances 331 were small for all sample sizes. In addition, when considering the expected lengths of all methods 332 which is shown in Table 2, these were wide in cases of $\sigma^{2}=2.0$ and became narrower when the 333 sample size increased, although they corresponded with the coverage probabilities in almost all 334 cases. Furthermore, the values were similar for all of the methods. Moreover, the expected lengths 335 of the intervals when $n=50, \delta=0.2$, and $\sigma^{2}=2.0$ were very much larger than the other cases 336 because the number of expected non-zero observations was small together with a large variance. 337 This case might have affected the parameter estimation, thus it is possible that the efficacy of the 338 confidence intervals constructed from it was not very good. 
Table 1. The coverage probabilities of $95 \%$ two-sided confidence intervals for a single coefficient of variation with the delta-lognormal

\begin{tabular}{|c|c|c|c|c|c|c|c|c|c|}
\hline \multirow{3}{*}{$n$} & \multirow{3}{*}{$\delta$} & \multirow{3}{*}{$\sigma^{2}$} & \multicolumn{7}{|c|}{ Coverage probabilities } \\
\hline & & & \multicolumn{3}{|c|}{ Equitailed confidence intervals } & \multicolumn{3}{|c|}{ HPD intervals } & \multirow[b]{2}{*}{ FGCI } \\
\hline & & & $\begin{array}{l}\text { Independent } \\
\text { Jeffreys }\end{array}$ & Jeffreys' Rule & Uniform & $\begin{array}{l}\text { Independent } \\
\text { Jeffreys }\end{array}$ & Jeffreys' Rule & Uniform & \\
\hline \multirow[t]{12}{*}{25} & 0.5 & 0.1 & 0.9600 & 0.9397 & 0.9647 & 0.9413 & 0.9181 & 0.9475 & 0.8686 \\
\hline & & 0.5 & 0.9718 & 0.9595 & 0.9763 & 0.9526 & 0.9308 & 0.9591 & 0.9415 \\
\hline & & 1.0 & 0.9593 & 0.9481 & 0.9668 & 0.9381 & 0.9187 & 0.9461 & 0.9518 \\
\hline & & 2.0 & 0.9521 & 0.9438 & 0.9608 & 0.9421 & 0.9309 & 0.9505 & 0.9523 \\
\hline & 0.8 & 0.1 & 0.9721 & 0.9657 & 0.9848 & 0.9539 & 0.9446 & 0.9746 & 0.9192 \\
\hline & & 0.5 & 0.9677 & 0.9618 & 0.9733 & 0.9579 & 0.9498 & 0.9686 & 0.9541 \\
\hline & & 1.0 & 0.9541 & 0.9487 & 0.9601 & 0.9499 & 0.9413 & 0.9589 & 0.9512 \\
\hline & & 2.0 & 0.9533 & 0.9482 & 0.9583 & 0.9482 & 0.9407 & 0.9551 & 0.9506 \\
\hline & 0.9 & 0.1 & 0.9669 & 0.9610 & 0.9960 & 0.9439 & 0.9391 & 0.9865 & 0.9393 \\
\hline & & 0.5 & 0.9607 & 0.9553 & 0.9683 & 0.9565 & 0.9500 & 0.9697 & 0.9559 \\
\hline & & 1.0 & 0.9511 & 0.9463 & 0.9573 & 0.9521 & 0.9464 & 0.9618 & 0.9539 \\
\hline & & 2.0 & 0.9524 & 0.9467 & 0.9563 & 0.9532 & 0.9475 & 0.9596 & 0.9481 \\
\hline \multirow[t]{14}{*}{50} & 0.2 & 0.1 & 0.9622 & 0.9349 & 0.9569 & 0.9384 & 0.9029 & 0.9289 & 0.8687 \\
\hline & & 0.5 & 0.9741 & 0.9547 & 0.9729 & 0.9539 & 0.9269 & 0.9517 & 0.9403 \\
\hline & & 1.0 & 0.9641 & 0.9477 & 0.9684 & 0.9449 & 0.9175 & 0.9477 & 0.9499 \\
\hline & & 2.0 & 0.9553 & 0.9447 & 0.9641 & 0.9402 & 0.9203 & 0.9485 & 0.9491 \\
\hline & 0.5 & 0.1 & 0.9605 & 0.9476 & 0.9619 & 0.9471 & 0.9301 & 0.9504 & 0.8694 \\
\hline & & 0.5 & 0.9669 & 0.9579 & 0.9689 & 0.9563 & 0.9435 & 0.9586 & 0.9356 \\
\hline & & 1.0 & 0.9585 & 0.9521 & 0.9622 & 0.9446 & 0.9329 & 0.9490 & 0.9499 \\
\hline & & 2.0 & 0.9534 & 0.9485 & 0.9575 & 0.9426 & 0.9346 & 0.9462 & 0.9537 \\
\hline & 0.8 & 0.1 & 0.9651 & 0.9600 & 0.9755 & 0.9508 & 0.9436 & 0.9659 & 0.9018 \\
\hline & & 0.5 & 0.9623 & 0.9581 & 0.9671 & 0.9547 & 0.9486 & 0.9621 & 0.9495 \\
\hline & & 1.0 & 0.9557 & 0.9523 & 0.9590 & 0.9467 & 0.9421 & 0.9527 & 0.9523 \\
\hline & & 2.0 & 0.9551 & 0.9529 & 0.9574 & 0.9525 & 0.9488 & 0.9556 & 0.9487 \\
\hline & 0.9 & 0.1 & 0.9660 & 0.9640 & 0.9830 & 0.9515 & 0.9463 & 0.9720 & 0.9274 \\
\hline & & 0.5 & 0.9581 & 0.9555 & 0.9623 & 0.9543 & 0.9509 & 0.9621 & 0.9537 \\
\hline
\end{tabular}




\begin{tabular}{|c|c|c|c|c|c|c|c|c|c|}
\hline \multirow{3}{*}{$n$} & \multirow{3}{*}{$\delta$} & \multirow{3}{*}{$\sigma^{2}$} & \multicolumn{7}{|c|}{ Coverage probabilities } \\
\hline & & & \multicolumn{3}{|c|}{ Equitailed confidence intervals } & \multicolumn{3}{|c|}{ HPD intervals } & \multirow[b]{2}{*}{ FGCI } \\
\hline & & & $\begin{array}{c}\text { Independent } \\
\text { Jeffreys }\end{array}$ & Jeffreys' Rule & Uniform & $\begin{array}{c}\text { Independent } \\
\text { Jeffreys }\end{array}$ & Jeffreys' Rule & Uniform & \\
\hline \multirow[t]{2}{*}{50} & 0.9 & 1.0 & 0.9519 & 0.9496 & 0.9554 & 0.9474 & 0.9443 & 0.9535 & 0.9535 \\
\hline & & 2.0 & 0.9507 & 0.9484 & 0.9537 & 0.9483 & 0.9450 & 0.9518 & 0.9501 \\
\hline \multirow[t]{16}{*}{100} & 0.2 & 0.1 & 0.9571 & 0.9355 & 0.9513 & 0.9406 & 0.9155 & 0.9325 & 0.8582 \\
\hline & & 0.5 & 0.9673 & 0.9532 & 0.9655 & 0.9539 & 0.9350 & 0.9509 & 0.9288 \\
\hline & & 1.0 & 0.9612 & 0.9519 & 0.9623 & 0.9433 & 0.9263 & 0.9430 & 0.9461 \\
\hline & & 2.0 & 0.9511 & 0.9435 & 0.9563 & 0.9401 & 0.9279 & 0.9434 & 0.9491 \\
\hline & 0.5 & 0.1 & 0.9578 & 0.9462 & 0.9587 & 0.9473 & 0.9356 & 0.9487 & 0.8591 \\
\hline & & 0.5 & 0.9605 & 0.9531 & 0.9621 & 0.9531 & 0.9432 & 0.9543 & 0.9315 \\
\hline & & 1.0 & 0.9566 & 0.9532 & 0.9585 & 0.9433 & 0.9367 & 0.9455 & 0.9468 \\
\hline & & 2.0 & 0.9546 & 0.9518 & 0.9559 & 0.9457 & 0.9412 & 0.9472 & 0.9516 \\
\hline & 0.8 & 0.1 & 0.9603 & 0.9566 & 0.9694 & 0.9464 & 0.9413 & 0.9578 & 0.8845 \\
\hline & & 0.5 & 0.9605 & 0.9580 & 0.9641 & 0.9461 & 0.9425 & 0.9508 & 0.9442 \\
\hline & & 1.0 & 0.9533 & 0.9505 & 0.9544 & 0.9485 & 0.9473 & 0.9517 & 0.9514 \\
\hline & & 2.0 & 0.9509 & 0.9499 & 0.9524 & 0.9476 & 0.9458 & 0.9499 & 0.9509 \\
\hline & 0.9 & 0.1 & 0.9657 & 0.9633 & 0.9768 & 0.9495 & 0.9461 & 0.9667 & 0.9065 \\
\hline & & 0.5 & 0.9538 & 0.9533 & 0.9582 & 0.9544 & 0.9509 & 0.9601 & 0.9473 \\
\hline & & 1.0 & 0.9534 & 0.9507 & 0.9539 & 0.9491 & 0.9475 & 0.9529 & 0.9491 \\
\hline & & 2.0 & 0.9505 & 0.9493 & 0.9527 & 0.9498 & 0.9480 & 0.9509 & 0.9512 \\
\hline \multirow[t]{8}{*}{200} & 0.2 & 0.1 & 0.9565 & 0.9407 & 0.9513 & 0.9425 & 0.9263 & 0.9381 & 0.8541 \\
\hline & & 0.5 & 0.9591 & 0.9473 & 0.9570 & 0.9485 & 0.9355 & 0.9461 & 0.9189 \\
\hline & & 1.0 & 0.9577 & 0.9496 & 0.9577 & 0.9406 & 0.9281 & 0.9397 & 0.9423 \\
\hline & & 2.0 & 0.9561 & 0.9515 & 0.9567 & 0.9399 & 0.9326 & 0.9417 & 0.9477 \\
\hline & 0.5 & 0.1 & 0.9564 & 0.9490 & 0.9573 & 0.9463 & 0.9389 & 0.9483 & 0.8567 \\
\hline & & 0.5 & 0.9565 & 0.9517 & 0.9575 & 0.9507 & 0.9440 & 0.9508 & 0.9249 \\
\hline & & 1.0 & 0.9555 & 0.9531 & 0.9560 & 0.9433 & 0.9397 & 0.9439 & 0.9438 \\
\hline & & 2.0 & 0.9541 & 0.9521 & 0.9552 & 0.9445 & 0.9408 & 0.9461 & 0.9469 \\
\hline
\end{tabular}


Table 1. Continued

345

\begin{tabular}{|c|c|c|c|c|c|c|c|c|c|}
\hline \multirow{3}{*}{$n$} & \multirow{3}{*}{$\delta$} & \multirow{3}{*}{$\sigma^{2}$} & \multicolumn{7}{|c|}{ Coverage probabilities } \\
\hline & & & \multicolumn{3}{|c|}{ Equitailed confidence intervals } & \multicolumn{3}{|c|}{ HPD intervals } & \multirow[b]{2}{*}{ FGCI } \\
\hline & & & $\begin{array}{c}\text { Independent } \\
\text { Jeffreys }\end{array}$ & Jeffreys' Rule & Uniform & $\begin{array}{l}\text { Independent } \\
\text { Jeffreys }\end{array}$ & Jeffreys' Rule & Uniform & \\
\hline \multirow[t]{8}{*}{200} & 0.8 & 0.1 & 0.9575 & 0.9537 & 0.9625 & 0.9509 & 0.9454 & 0.9575 & 0.8771 \\
\hline & & 0.5 & 0.9555 & 0.9531 & 0.9578 & 0.9506 & 0.9476 & 0.9536 & 0.9409 \\
\hline & & 1.0 & 0.9517 & 0.9503 & 0.9523 & 0.9475 & 0.9465 & 0.9497 & 0.9503 \\
\hline & & 2.0 & 0.9507 & 0.9500 & 0.9513 & 0.9464 & 0.9457 & 0.9473 & 0.9527 \\
\hline & 0.9 & 0.1 & 0.9603 & 0.9587 & 0.9693 & 0.9495 & 0.9465 & 0.9601 & 0.8949 \\
\hline & & 0.5 & 0.9541 & 0.9527 & 0.9565 & 0.9482 & 0.9463 & 0.9505 & 0.9445 \\
\hline & & 1.0 & 0.9523 & 0.9518 & 0.9531 & 0.9462 & 0.9444 & 0.9468 & 0.9480 \\
\hline & & 2.0 & 0.9513 & 0.9513 & 0.9517 & 0.9514 & 0.9511 & 0.9523 & 0.9500 \\
\hline
\end{tabular}

346

347

348

349

Table 2. The expected lengths of $95 \%$ two-sided confidence intervals for a single coefficient of variation with the delta-lognormal distribution

\begin{tabular}{|c|c|c|c|c|c|c|c|c|c|}
\hline \multirow{3}{*}{$n$} & \multirow{3}{*}{$\delta$} & \multirow{3}{*}{$\sigma^{2}$} & \multicolumn{7}{|c|}{ Expected lengths } \\
\hline & & & \multicolumn{3}{|c|}{ Equitailed confidence intervals } & \multicolumn{3}{|c|}{ HPD intervals } & \multirow[b]{2}{*}{ FGCI } \\
\hline & & & $\begin{array}{c}\text { Independent } \\
\text { Jeffreys }\end{array}$ & Jeffreys' Rule & Uniform & $\begin{array}{c}\text { Independent } \\
\text { Jeffreys }\end{array}$ & Jeffreys' Rule & Uniform & \\
\hline \multirow[t]{10}{*}{25} & 0.5 & 0.1 & 0.7297 & 0.6901 & 0.7259 & 0.7126 & 0.6750 & 0.7087 & 0.5254 \\
\hline & & 0.5 & 1.5150 & 1.4019 & 1.6064 & 1.3664 & 1.2766 & 1.4273 & 1.3969 \\
\hline & & 1.0 & 4.2513 & 3.8066 & 4.7342 & 3.2613 & 2.9912 & 3.5216 & 4.1004 \\
\hline & & 2.0 & 33.7300 & 27.5715 & 42.9352 & 17.2204 & 14.9908 & 19.8622 & 32.8632 \\
\hline & 0.8 & 0.1 & 0.4319 & 0.4176 & 0.4390 & 0.4222 & 0.4084 & 0.4296 & 0.3280 \\
\hline & & 0.5 & 0.8803 & 0.8469 & 0.9149 & 0.8168 & 0.7885 & 0.8457 & 0.8324 \\
\hline & & 1.0 & 2.1046 & 2.0054 & 2.2163 & 1.7993 & 1.7294 & 1.8798 & 2.0750 \\
\hline & & 2.0 & 9.4926 & 8.8318 & 10.2578 & 6.9057 & 6.5518 & 7.3427 & 9.4872 \\
\hline & 0.9 & 0.1 & 0.3346 & 0.3248 & 0.3518 & 0.3232 & 0.3139 & 0.3410 & 0.2710 \\
\hline & & 0.5 & 0.7577 & 0.7342 & 0.7880 & 0.7082 & 0.6881 & 0.7346 & 0.7376 \\
\hline
\end{tabular}




\begin{tabular}{|c|c|c|c|c|c|c|c|c|c|}
\hline \multirow{3}{*}{$n$} & \multirow{3}{*}{$\delta$} & \multirow{3}{*}{$\sigma^{2}$} & \multicolumn{7}{|c|}{ Expected lengths } \\
\hline & & & \multicolumn{3}{|c|}{ Equitailed confidence intervals } & \multicolumn{3}{|c|}{ HPD intervals } & \multirow[b]{2}{*}{ FGCI } \\
\hline & & & $\begin{array}{l}\text { Independent } \\
\text { Jeffreys }\end{array}$ & Jeffreys' Rule & Uniform & $\begin{array}{l}\text { Independent } \\
\text { Jeffreys }\end{array}$ & Jeffreys' Rule & Uniform & \\
\hline \multirow[t]{2}{*}{25} & 0.9 & 1.0 & 1.7631 & 1.6978 & 1.8460 & 1.5534 & 1.5049 & 1.6173 & 1.7591 \\
\hline & & 2.0 & 7.4883 & 7.0855 & 7.9907 & 5.8011 & 5.5645 & 6.1147 & 7.4510 \\
\hline \multirow[t]{16}{*}{50} & 0.2 & 0.1 & 1.3339 & 1.2287 & 1.3061 & 1.2863 & 1.1889 & 1.2586 & 0.9404 \\
\hline & & 0.5 & 3.0407 & 2.6749 & 3.3153 & 2.5845 & 2.3218 & 2.7216 & 2.7064 \\
\hline & & 1.0 & 10.4439 & 8.5127 & 12.8712 & 6.8066 & 5.8704 & 7.6676 & 9.7812 \\
\hline & & 2.0 & 218.3340 & 123.9037 & 409.4769 & 77.4546 & 55.6155 & 141.4522 & 517.7059 \\
\hline & 0.5 & 0.1 & 0.5278 & 0.5128 & 0.5246 & 0.5204 & 0.5059 & 0.5174 & 0.3787 \\
\hline & & 0.5 & 0.9170 & 0.8878 & 0.9311 & 0.8739 & 0.8476 & 0.8849 & 0.8188 \\
\hline & & 1.0 & 2.0182 & 1.9446 & 2.0759 & 1.8142 & 1.7545 & 1.8557 & 1.9373 \\
\hline & & 2.0 & 7.8105 & 7.4368 & 8.1546 & 6.3278 & 6.0759 & 6.5403 & 7.9104 \\
\hline & 0.8 & 0.1 & 0.3063 & 0.3012 & 0.3082 & 0.3023 & 0.2972 & 0.3043 & 0.2298 \\
\hline & & 0.5 & 0.5519 & 0.5429 & 0.5603 & 0.5349 & 0.5265 & 0.5427 & 0.5203 \\
\hline & & 1.0 & 1.1725 & 1.1514 & 1.1951 & 1.0952 & 1.0773 & 1.1143 & 1.1529 \\
\hline & & 2.0 & 3.9949 & 3.9093 & 4.0925 & 3.5027 & 3.4371 & 3.5760 & 3.9755 \\
\hline & 0.9 & 0.1 & 0.2436 & 0.2400 & 0.2491 & 0.2390 & 0.2355 & 0.2447 & 0.1923 \\
\hline & & 0.5 & 0.4867 & 0.4799 & 0.4948 & 0.4717 & 0.4655 & 0.4793 & 0.4729 \\
\hline & & 1.0 & 1.0405 & 1.0248 & 1.0588 & 0.9804 & 0.9674 & 0.9971 & 1.7590 \\
\hline & & 2.0 & 3.4585 & 3.3987 & 3.5352 & 3.0534 & 3.0069 & 3.1113 & 3.4754 \\
\hline \multirow[t]{8}{*}{100} & 0.2 & 0.1 & 0.9341 & 0.8971 & 0.9200 & 0.9190 & 0.8829 & 0.9050 & 0.6628 \\
\hline & & 0.5 & 1.6376 & 1.5617 & 1.6550 & 1.5542 & 1.4859 & 1.5640 & 1.4196 \\
\hline & & 1.0 & 3.7403 & 3.5325 & 3.8579 & 3.2758 & 3.1139 & 3.3492 & 3.5025 \\
\hline & & 2.0 & 16.4708 & 15.2363 & 17.4752 & 12.4579 & 11.7196 & 12.9891 & 15.9392 \\
\hline & 0.5 & 0.1 & 0.3774 & 0.3719 & 0.3760 & 0.3738 & 0.3685 & 0.3725 & 0.2712 \\
\hline & & 0.5 & 0.6066 & 0.5975 & 0.6096 & 0.5957 & 0.5871 & 0.5986 & 0.5354 \\
\hline & & 1.0 & 1.2286 & 1.2092 & 1.2409 & 1.1698 & 1.1521 & 1.1801 & 1.1719 \\
\hline & & 2.0 & 3.9868 & 3.9149 & 4.0450 & 3.6197 & 3.5622 & 3.6637 & 3.9565 \\
\hline
\end{tabular}


Table 2. Continued

353

\begin{tabular}{|c|c|c|c|c|c|c|c|c|c|}
\hline \multirow{3}{*}{$n$} & \multirow{3}{*}{$\delta$} & \multirow{3}{*}{$\sigma^{2}$} & \multicolumn{7}{|c|}{ Expected lengths } \\
\hline & & & \multicolumn{3}{|c|}{ Equitailed confidence intervals } & \multicolumn{3}{|c|}{ HPD intervals } & \multirow[b]{2}{*}{ FGCI } \\
\hline & & & $\begin{array}{l}\text { Independent } \\
\text { Jeffreys }\end{array}$ & Jeffreys' Rule & Uniform & $\begin{array}{c}\text { Independent } \\
\text { Jeffreys }\end{array}$ & Jeffreys' Rule & Uniform & \\
\hline \multirow[t]{8}{*}{100} & 0.8 & 0.1 & 0.2190 & 0.2172 & 0.2196 & 0.2171 & 0.2153 & 0.2177 & 0.1633 \\
\hline & & 0.5 & 0.3732 & 0.3703 & 0.3758 & 0.3664 & 0.3637 & 0.3689 & 0.3487 \\
\hline & & 1.0 & 0.7565 & 0.7502 & 0.7625 & 0.7330 & 0.7274 & 0.7388 & 0.7426 \\
\hline & & 2.0 & 2.3285 & 2.3082 & 2.3515 & 2.1759 & 2.1582 & 2.1948 & 2.3232 \\
\hline & 0.9 & 0.1 & 0.1743 & 0.1729 & 0.1761 & 0.1723 & 0.1710 & 0.1742 & 0.1358 \\
\hline & & 0.5 & 0.3296 & 0.3275 & 0.3322 & 0.3236 & 0.3216 & 0.3262 & 0.3182 \\
\hline & & 1.0 & 0.6764 & 0.6720 & 0.6817 & 0.6560 & 0.6521 & 0.6612 & 0.6717 \\
\hline & & 2.0 & 2.0505 & 2.0360 & 2.0685 & 1.9451 & 1.9328 & 1.9618 & 2.0511 \\
\hline \multirow[t]{16}{*}{200} & 0.2 & 0.1 & 0.6714 & 0.6576 & 0.6658 & 0.6638 & 0.6502 & 0.6582 & 0.4777 \\
\hline & & 0.5 & 1.0731 & 1.0499 & 1.0742 & 1.0478 & 1.0255 & 1.0481 & 0.9122 \\
\hline & & 1.0 & 2.1624 & 2.1109 & 2.1802 & 2.0517 & 2.0062 & 2.0653 & 2.0429 \\
\hline & & 2.0 & 7.4107 & 7.2073 & 7.5181 & 6.5125 & 6.3549 & 6.5886 & 7.2846 \\
\hline & 0.5 & 0.1 & 0.2704 & 0.2684 & 0.2699 & 0.2685 & 0.2666 & 0.2680 & 0.1946 \\
\hline & & 0.5 & 0.4194 & 0.4163 & 0.4202 & 0.4154 & 0.4123 & 0.4161 & 0.3676 \\
\hline & & 1.0 & 0.8190 & 0.8128 & 0.8224 & 0.7971 & 0.7910 & 0.8001 & 0.7794 \\
\hline & & 2.0 & 2.4764 & 2.4567 & 2.4900 & 2.3551 & 2.3375 & 2.3669 & 2.4492 \\
\hline & 0.8 & 0.1 & 0.1566 & 0.1559 & 0.1568 & 0.1557 & 0.1550 & 0.1558 & 0.1163 \\
\hline & & 0.5 & 0.2587 & 0.2577 & 0.2595 & 0.2562 & 0.2552 & 0.2569 & 0.2415 \\
\hline & & 1.0 & 0.5145 & 0.5127 & 0.5166 & 0.5055 & 0.5036 & 0.5073 & 0.5045 \\
\hline & & 2.0 & 1.5141 & 1.5085 & 1.5205 & 1.4683 & 1.4623 & 1.4744 & 1.5080 \\
\hline & 0.9 & 0.1 & 0.1247 & 0.1243 & 0.1254 & 0.1238 & 0.1233 & 0.1244 & 0.0965 \\
\hline & & 0.5 & 0.2283 & 0.2276 & 0.2292 & 0.2260 & 0.2254 & 0.2268 & 0.2200 \\
\hline & & 1.0 & 0.4617 & 0.4602 & 0.4635 & 0.4521 & 0.4508 & 0.4539 & 0.4557 \\
\hline & & 2.0 & 1.3467 & 1.3423 & 1.3523 & 1.3067 & 1.3028 & 1.3121 & 1.3467 \\
\hline
\end{tabular}

354 


\section{An empirical studies}

356 Ananthakrishnan and Soman (1989) studied a daily rainfall data series focusing on the normalized rainfall curve (NRC). They found that the NRC is uniquely determined by the coefficient of variation of the rainfall series. To verify the effectiveness of the proposed confidence intervals, we used two examples of rainfall datasets from Nan province, Thailand as follows.

\section{Example 1}

The rainfall data was collected in July 2015 for national parks in Nan province, Thailand: Doi Phu Kha, Mae Charim, Nanthaburi, Tham Sa Koen, Sri Nan, Khun Sathan, and Doi Pha Klong recorded by the Protected Area Regional Office 13 Phrae, Thailand. For this data series, there were 217 rainfall measurements, of which 117 were positive, showing a right-skewed distribution. The density of this data is presented in Fig. 1. Next, the minimum Akaike information criterion (AIC) was first to test the distribution of the positive rainfall data. The results in Table 3 reveal that the AIC value of the lognormal distribution was smallest, thus the distribution of this positive data series was the lognormal distribution. To validate the AIC test, a normal Q-Q plot for logtransformation data series is shown in Fig. 2. The distribution of zero values in this rainfall series coincided with the method in Section 2 for a binomial distribution. Therefore, a delta-lognormal distribution was appropriate for these data. Next, summary statistics were computed: $n=217$, $\hat{\delta}=0.5392, \hat{\mu}=2.4762, \hat{\sigma}^{2}=0.9381$, and $C V=1.9337$. Finally, the 95\% confidence intervals for $\eta$ were calculated, as reported in Table 4 . These results correspond with those from the simulation study when the sample size was large in that the coverage probabilities of the Bayesian methods (equitailed confidence intervals) were greater than the target. This indicates that the Bayesian method using the Jeffreys' Rule prior is appropriate to construct a confidence interval for this rainfall data due to it having the shortest expected length compared to the other methods. The estimated coefficient of variation in Table 4 means that the variability of the rainfall was rather high. This indicates that the rainfall fluctuated, which would have affected the water levels in the area and there could have been flooding, which would have affected agricultural productivity in the area.

Table 3. AIC results to check the distributions of positive rainfall values in July 2015 for national parks in Nan province, Thailand

\begin{tabular}{lcccc}
\hline Densities & Normal & Lognormal & Cauchy & Exponential \\
\hline AIC & 978.4592 & $\mathbf{9 0 6 . 9 9 0 3}$ & 971.7420 & 908.4876 \\
\hline
\end{tabular}




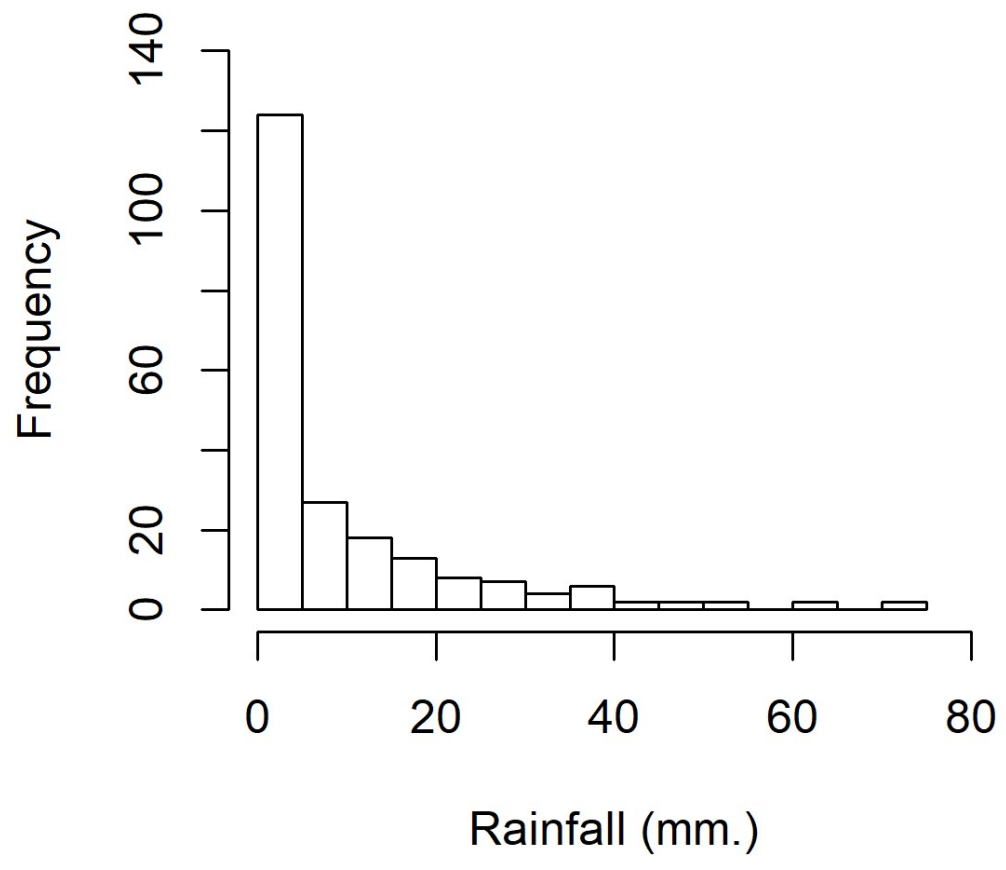

389 Figure 1. The density of rainfall data in July 2015 for national parks in Nan province, Thailand 390

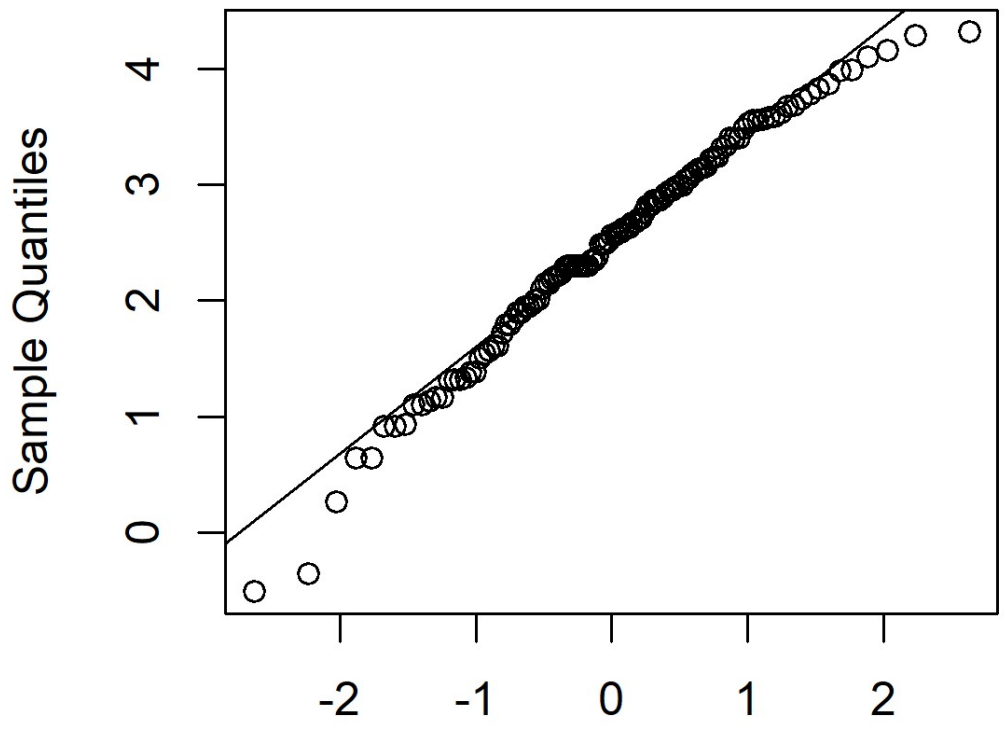

Figure 2. The normal Q-Q plot of log-transformed for positive rainfall data in July 2015 for 395 national parks in Nan province, Thailand 
Table 4. The $95 \%$ confidence intervals for a single coefficient of variation of rainfall data in July 2015 for national parks in Nan province, Thailand

\begin{tabular}{lccc}
\hline \multicolumn{1}{c}{ Methods } & \multicolumn{2}{c}{ Confidence intervals for $\eta$} & $\begin{array}{c}\text { Length of } \\
\text { Intervals }\end{array}$ \\
\cline { 2 - 3 } & Lower & Upper & 0.7009 \\
Bayesian: Independent Jeffreys' (Equitailed) & 1.6570 & 2.3579 & 0.6850 \\
Bayesian: Jeffreys' Rule (Equitailed) & 1.6610 & 2.3460 & 0.6914 \\
Bayesian: Uniform (Equitailed) & 1.6646 & 2.3560 & 0.6852 \\
Bayesian: Independent Jeffreys' (HPD) & 1.6314 & 2.3166 & 0.6746 \\
Bayesian: Jeffreys' Rule (HPD) & 1.6424 & 2.3170 & 0.6796 \\
Bayesian: Uniform (HPD) & 1.6549 & 2.3345 & 0.6506 \\
FGCI & 1.6788 & 2.3294 & \\
\hline
\end{tabular}

\section{Example 2}

To investigate variation in rainfall, a rainfall dataset reported by the Upper Northern Region Irrigation Hydrology Center, Bureau of Water Management and Hydrology Royal Irrigation Department Thailand for August 2018 comprising eight precipitation stations in Nan province, Thailand (Muang, Thawangpha, Thung Chang, Pua, Song Khwae, Santisuk, Chaloem Phra Kiat, and Chiang Klang) was used. There were 248 observed values comprising 91 zero values and 157 positive values; the density of this rainfall data is shown in Fig. 3. The positive values follow a lognormal distribution, as indicated by the minimum AIC in Table 5 and a normal Q-Q plot of the log-transformed data displayed in Fig. 4. In addition, the zero values have a binomial distribution (as discussed by Aitchison (1955)), thus the overall distribution is delta-lognormal. The summary statistics were $n=248, \hat{\delta}=0.6331, \hat{\mu}=1.5822, \hat{\sigma}^{2}=2.2598$, and $C V=3.7595$.

The results in Table 6 report the 95\% confidence intervals for $\eta$. The results of the methods to construct the confidence intervals are in accordance with those in the simulation study for the case of a large sample size. The Bayesian method based on the Jeffreys' Rule prior (equitailed confidence intervals) had the shortest expected length. The coefficient of variation estimation in Table 6 indicates that the rainfall of this area was highly volatile, which affected the water level of the Nan River. Moreover, there might have been flooding in some of the areas due to high rainfall.

Table 5. AIC results to check the distributions of positive rainfall values in August 2018 from eight precipitation stations in Nan province, Thailand

\begin{tabular}{lcccc}
\hline Densities & Normal & Lognormal & Cauchy & Exponential \\
\hline AIC & $1,596.0140$ & $\mathbf{1 , 0 7 3 . 3 3 8 0}$ & $1,196.0190$ & $1,186.0920$ \\
\hline
\end{tabular}




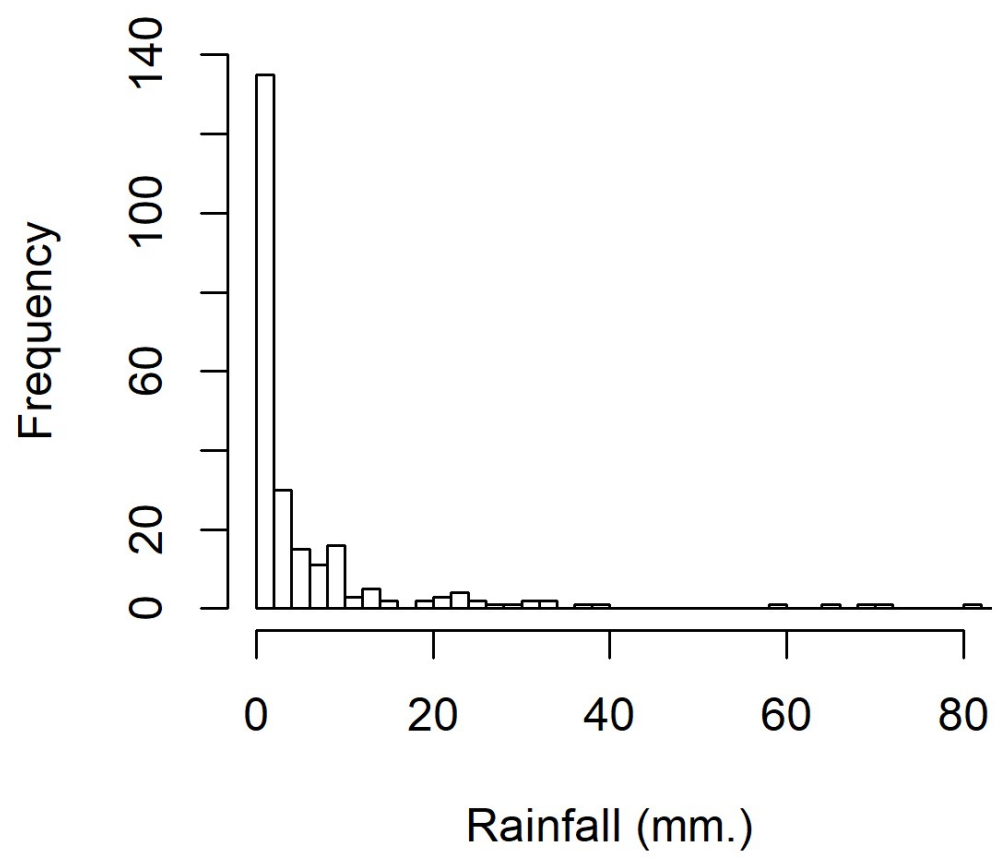

423

424 425 426

427 428 429
Figure 3. The density of rainfall data in August 2018 from eight precipitation stations in Nan province, Thailand

Table 6. The $95 \%$ confidence intervals for a single coefficient of variation of rainfall data in August 2018 from eight precipitation stations in Nan province, Thailand

\begin{tabular}{lccc}
\hline \multirow{2}{*}{ Methods } & \multicolumn{2}{c}{ Confidence intervals for $\eta$} & $\begin{array}{c}\text { Length of } \\
\text { Intervals }\end{array}$ \\
\cline { 2 - 3 } & Lower & Upper & 2.2567 \\
\hline Bayesian: Independent Jeffreys' (Equitailed) & 2.9429 & 5.1996 & 2.1675 \\
Bayesian: Jeffreys' Rule (Equitailed) & 2.9536 & 5.1211 & 2.2412 \\
Bayesian: Uniform (Equitailed) & 2.9784 & 5.2196 & 2.1456 \\
Bayesian: Independent Jeffreys' (HPD) & 2.7824 & 4.9280 & 2.0870 \\
Bayesian: Jeffreys' Rule (HPD) & 2.8144 & 4.9014 & 2.1452 \\
Bayesian: Uniform (HPD) & 2.8704 & 5.0156 & 2.1496 \\
FGCI & 2.9795 & 5.1291 & \\
\hline
\end{tabular}




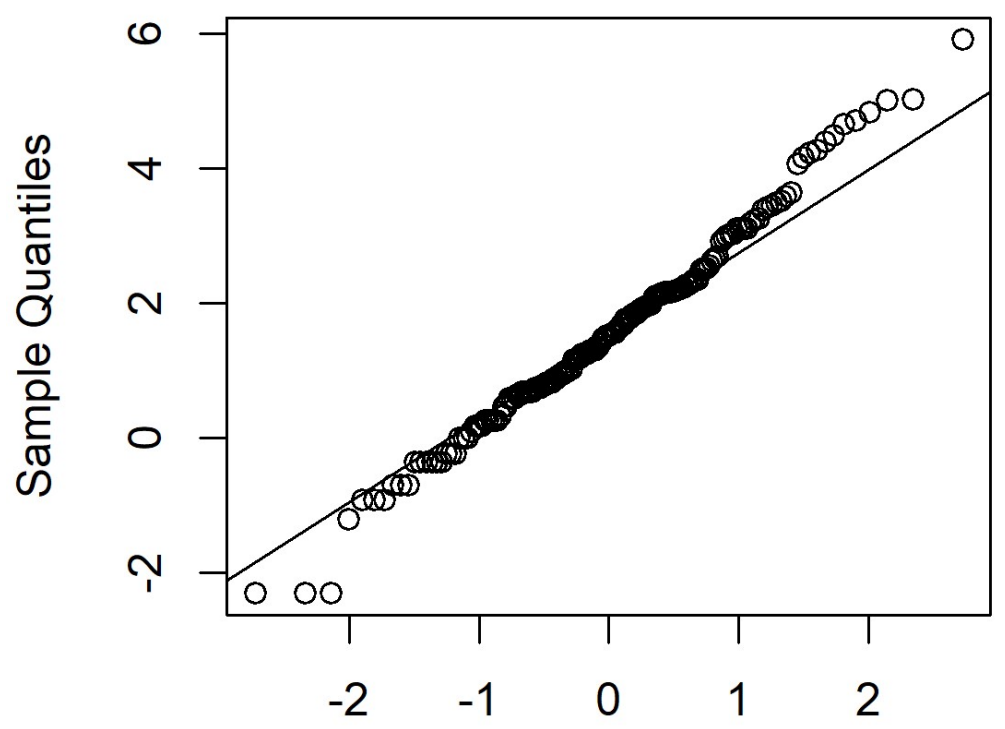

Figure 4. The normal Q-Q plot of log-transformed for positive rainfall data in August 2018

\section{Discussion}

Our findings reveal that the Bayesian method using the independent Jeffreys' prior to construct the equitailed confidence intervals performed well for all cases due to the coverage probabilities being consistently greater than or close to the nominal confidence level while the expected lengths were mostly no different from the other methods. Moreover, underestimation occurred for a few of the cases when applying the Bayesian methods based on the Jeffreys' Rule prior (equitailed), the independence Jeffreys' prior (HPD), and the uniform prior (HPD), and it appeared in almost all cases of the Jeffreys' Rule prior (HPD). In contrast, overestimation occurred in a few cases of applying the Bayesian method based on the uniform prior (equitailed) when the sample size was less than 100 together with a small variance and high proportion of non-zero values.

\section{Conclusions}

We proposed the construction of confidence intervals for a single coefficient of variation of a deltalognormal distribution using Bayesian methods and compared them with FGCI. The Bayesian methods, which are based on the independent Jeffreys' prior, the Jeffreys' Rule prior, and the uniform prior, were constructed under equitailed confidence intervals or HPD intervals. The performance of the confidence intervals was assessed using the coverage probability and expected length through Monte Carlo simulations. The simulation studies showed that the Bayesian equitailed confidence intervals based on the independent Jeffreys' prior is recommended as a confidence interval for a single coefficient of variation. Future researchers may also be extended to the case of the coefficients of variation function. 


\section{References}

457 Aitchison J. 1955. On the distribution of a positive random variable having a discrete probability 458 mass at the origin. Journal of the American Statistical Association 50:901-908 DOI: $45910.2307 / 2281175$.

460 Ananthakrishnan R, Soman MK. 1989. Statistical distribution of daily rainfall and its association 461 with the coefficient of variation of rainfall series. International Journal of Climatology 9:485-500. 462 Bolstad WM, Curran JM. 2017. Introduction to Bayesian statistics. Hoboken, New Jersey: John 463 Wiley \& Sons.

464 Buntao N, Niwitpong S. 2012. Confidence intervals for the difference of coefficients of variation 465 for lognormal distributions and delta-lognormal distributions. Applied Mathematical Sciences 6:6691-6704.

Buntao N, Niwitpong S. 2013. Confidence intervals for the ratio of coefficients of variation of delta-lognormal distribution. Applied Mathematical Sciences 7:3811-3818. Callahan CM, Kesterson JG, Tierney WM. 1997. Association of symptoms of depression with diagnostic test charges among older adults. Annals of Internal Medicine 126:426-432. Chen Y-H, Zhou X-H. 2006. Generalized confidence intervals for the ratio or difference of two means for lognormal populations with zeros. UW Biostatistics Working Paper Series. Climatological group. 2015. The climate of Thailand. Available at https://www.tmd.go.th/en/archive/thailand_climate.pdf (accessed 1 May 2019) D'Cunha JG, Rao KA. 2014. Bayesian inference for mean of the lognormal distribution. International Journal of Scientific and Research Publications 4. standard deviation. Statistical Methods in Medical Research 21:347-359 DOI: 10.1177/09622802 10383082.

Fisher RA. 1930. Inverse probability. Mathematical Proceedings of the Cambridge Philosophical Society 26:528-535 DOI: 10.1017/S0305004100016297. Fletcher D. 2008. Confidence intervals for the mean of the delta-lognormal distribution. Environmental and Ecological Statistics 15:175-189 DOI: 10.1007/s10651-007-0046-8.

Fukuchi H. 1988. Correlation properties of rainfall rates in the United Kingdom. IEE Proceedings $H$ - Microwaves, Antennas and Propagation 135:83-88 DOI: 10.1049/ip-h-2.1988.0018. Gulhar M, Kibria BMG, Albatineh AN, Ahmed NU. 2012. A comparison of some confidence intervals for estimating the population coefficient of variation: a simulation study. SORT-Statistics and Operations Research Transactions 36:45-68. Hannig J. 2009. On generalized fiducial inference. Statistica Sinica 19:491-544. Hannig J, E L, Abdel-Karim A, Iyer H. 2006a. Simultaneous fiducial generalized confidence intervals for ratios of means of lognormal distributions. Austrian Journal of Statistics 35:261-269. Hannig J, Iyer H, Patterson P. 2006b. Fiducial generalized confidence intervals. Journal of the American Statistical Association 101:254-269 DOI: 10.1198/016214505000000736. 
496

497

498

499

500

501

502

503

504

505

506

507

508

509

510

511

512

513

514

515

516

517

518

519

520

521

522

523

524

525

526

527

528

529

530

531

532

533

534

535

Harold Jeffreys, F.R.S. 1946. An invariant form for the prior probability in estimation problems. Proceedings of the Royal Society of London, Series A, Mathematical and Physical Sciences 186:453-461 DOI: 10.1098/rspa.1946.0056.

Harvey J, van der Merwe AJ. 2012. Bayesian confidence intervals for means and variances of lognormal and bivariate lognormal distributions. Journal of Statistical Planning and Inference 142:1294-1309 DOI: 10.1016/j.jspi.2011.12.006.

Hyndman RJ. 1995. Highest-density forecast regions for non-linear and non-normal time series models. Journal of Forecasting 14:431-441.

Kalkur T. A, Rao A. 2017. Bayes estimator for coefficient of variation and inverse coefficient of variation for the normal distribution. International Journal of Statistics and Systems 12:721-732. Kim YY, Lee JT, Choi GH. 2005. An investigation on the causes of cycle variation in direct injection hydrogen fueled engines. International Journal of Hydrogen Energy 30:69-76 DOI: 10.1016/j.ijhydene.2004.03.041.

Kong CY, Jamaludin S, Yusof F, Foo HM. 2012. Parameter estimation for bivariate mixed lognormal distribution. Journal of Science and Technology 4:41-48.

Kvanli AH, Shen YK, Deng LY. 1998. Construction of confidence intervals for the mean of a population containing many zero values. Journal of Business \& Economic Statistics 16:362-368 DOI: 10.1080/07350015.1998.10524776.

Lee PM. 2012. Bayesian statistics: an introduction. Chichester, West Sussex: John Wiley \& Sons. Li X, Zhou X, Tian L. 2013. Interval estimation for the mean of lognormal data with excess zeros. Statistics \& Probability Letters 83:2447-2453 DOI: 10.1016/j.spl.2013.07.004.

Mahmoudvand R, Hassani H. 2009. Two new confidence intervals for the coefficient of variation in a normal distribution. Journal of Applied Statistics 36:429-442 DOI: 10.1080/02664760802474 249.

Maneerat P, Niwitpong S, Niwitpong S. 2018. Confidence Intervals for the Ratio of Means of Delta-Lognormal Distribution. In: Anh LH, Dong LS, Kreinovich V, Thach NN, eds. Econometrics for Financial Applications. Studies in Computational Intelligence. Springer International Publishing, 161-174.

Niwitpong S. 2013. Confidence intervals for coefficient of variation of lognormal distribution with restricted parameter space. Applied Mathematical Sciences 7:3805-3810.

O'Reilly JX, Mars RB. 2015. Bayesian models in cognitive neuroscience: a tutorial. In: Forstmann BU, Wagenmakers E-J, eds. An Introduction to Model-Based Cognitive Neuroscience. Springer, 179-197.

Owen WJ, DeRouen TA. 1980. Estimation of the mean for lognormal data containing zeroes and left- censored values, with applications to the measurement of worker exposure to air contaminants. Biometrics 36:707-719 DOI: 10.2307/2556125.

Rao KA, D'Cunha JG. 2016. Bayesian inference for median of the lognormal distribution. Journal of Modern Applied Statistical Methods 15:526-535 DOI: 10.22237/jmasm/1478003400.

Rubio FJ, Liseo B. 2014. On the independence Jeffreys prior for skew-symmetric models. Statistics and Probability Letters 85:91-97. 
536 Sangnawakij P, Niwitpong S. 2017. Confidence intervals for coefficients of variation in twoparameter exponential distributions. Communications in Statistics - Simulation and Computation 46:6618-6630 DOI: 10.1080/03610918.2016.1208236. of variation of the gamma distributions. In: Huynh VN, Inuiguchi M, Demoeux T, eds. Integrated Uncertainty in Knowledge Modelling and Decision Making. Lecture Notes in Computer Science.

542 Springer Cham, 193-203.

543 Shimizu K. 1993. A bivariate mixed lognormal distribution with an analysis of rainfall data. 544 Journal of Applied Meteorology 32:161-171.

545 Stone JV. 2013. Bayes' Rule: a tutorial introduction to Bayesian analysis. Sheffield: Sebtel Press. 546 Thangjai W, Niwitpong S. 2017. Confidence intervals for the weighted coefficients of variation of 547 two-parameter exponential distributions. Cogent Mathematics 4:1315880 DOI: 10.1080/2331183 $548 \quad 5.2017 .1315880$.

549 Tian L. 2005. Inferences on the mean of zero-inflated lognormal data: the generalized variable approach. Statistics in Medicine 24:3223-3232 DOI: 10.1002/sim.2169.

551 Tian L, Wu J. 2006. Confidence intervals for the mean of lognormal data with excess zeros. Biometrical Journal. Biometrische Zeitschrift 48:149-156. van Zyl R, van der Merwe AJ. 2017. A Bayesian control chart for a common coefficient of variation. Communications in Statistics - Theory and Methods 46:5795-5811 DOI: 10.1080/03610 926.2015.1112914. Venables WN, Smith DM. 2009. An introduction to R. Bristol: Network Theory Ltd. Wong ACM, Wu J. 2002. Small sample asymptotic inference for the coefficient of variation: normal and nonnormal models. Journal of Statistical Planning and Inference 104:73-82 DOI: 10.1016/S0378-3758(01)00241-5. independent coefficients of variation of normal distribution. Far East Journal of Mathematical Sciences 98:741-757. lognormal distribution: an application to New Zealand trawl survey data. Journal of Applied Statistics 41:1471-1485 DOI: 10.1080/02664763.2014.881780. 11:95-102.

568 Yosboonruang N, Niwitpong S, Niwitpong S. 2018. Confidence intervals for the coefficient of variation of the delta-lognormal distribution. In: Anh LH, Dong LS, Kreinovich V, Thach NN, eds. Econometrics for Financial Applications. Studies in Computational Intelligence. Springer International Publishing, 327-337. Yosboonruang N, Niwitpong S, Niwitpong S. 2019. Confidence intervals for coefficient of variation of three parameters delta-lognormal distribution. In: Kreinovich V, Sriboonchitta S, eds. 
576 Yue S. 2000. The bivariate lognormal distribution to model a multivariate flood episode.

577 Hydrological Processes 14:2575-2588.

578 Zhou XH, Tu W. 2000. Confidence intervals for the mean of diagnostic test charge data containing 579 zeros. Biometrics 56:1118-1125.

580

581 Supplemental Files

582 Dataset 1: Rainfall data (mm.) in July 2015 for national parks in Nan province, Thailand: Doi 583 Phu Kha, Mae Charim, Nanthaburi, Tham Sa Koen, Sri Nan, Khun Sathan, and Doi Pha Klong 584 as shown in Dataset $\mathrm{S} 1$.

585 Dataset 2: Rainfall data (mm.) in August 2018 from eight precipitation stations in Nan province, 586 Thailand: Muang, Thawangpha, Thung Chang, Pua, Song Khwae, Santisuk, Chaloem Phra Kiat, 587 and Chiang Klang as shown in Dataset S2.

588

589 Dataset S1. Rainfall data (mm.) in July 2015 for national parks in Nan province, Thailand 590

\begin{tabular}{cccccccc}
\hline Date & NP1 & NP2 & NP3 & NP4 & NP5 & NP6 & NP7 \\
\hline 1 & 0 & 0 & 0 & 0 & 0 & 0 & 0 \\
2 & 0 & 0 & 0 & 0 & 0 & 0 & 0 \\
3 & 0 & 0 & 0 & 0 & 0 & 0 & 0 \\
4 & 0 & 0 & 0 & 0 & 0 & 0 & 0 \\
5 & 1.9 & 0 & 0 & 0 & 0 & 0 & 0 \\
6 & 47.9 & 0 & 0 & 0 & 0 & 0 & 0 \\
7 & 10.5 & 0 & 8.9 & 2.55 & 12.0 & 0 & 0 \\
8 & 43.8 & 3.0 & 3.7 & 39.5 & 46.0 & 8.6 & 4.5 \\
9 & 1.9 & 0 & 0.7 & 0 & 0 & 10.8 & 0 \\
10 & 0.6 & 0 & 10.5 & 0 & 0 & 0 & 0 \\
11 & 5.6 & 0 & 28.2 & 5.0 & 21.0 & 63.9 & 0 \\
12 & 0 & 0 & 25.7 & 27.5 & 0 & 0 & 0 \\
13 & 3.1 & 0 & 13.4 & 17.5 & 0 & 30.0 & 0 \\
14 & 3.2 & 0 & 0 & 0 & 0 & 9.4 & 0 \\
15 & 13.8 & 0 & 14.6 & 5.0 & 0 & 18.6 & 0 \\
16 & 3.0 & 0 & 7.0 & 17.5 & 6.0 & 8.6 & 10.0 \\
17 & 7.4 & 0 & 36.2 & 0 & 0 & 4.7 & 0 \\
18 & 14.5 & 0 & 3.2 & 3.75 & 0 & 2.5 & 0 \\
19 & 34.2 & 13.0 & 40.0 & 25.0 & 12.0 & 10.0 & 0 \\
20 & 14.9 & 0 & 16.7 & 17.5 & 0 & 4.0 & 10.0 \\
21 & 15.8 & 36.0 & 54.2 & 30.0 & 73.0 & 35.3 & 30.0 \\
22 & 3.8 & 0 & 4.8 & 2.5 & 7.0 & 6.3 & 0 \\
23 & 42.1 & 0 & 19.0 & 75.0 & 13.0 & 23.1 & 10.0 \\
24 & 23.2 & 0 & 9.1 & 16.8 & 0 & 13.9 & 0 \\
25 & 60.7 & 6.0 & 6.7 & 53.75 & 0 & 6.7 & 0 \\
26 & 23.7 & 10.0 & 9.8 & 37.5 & 0 & 25.5 & 0 \\
27 & 22.5 & 0 & 32.5 & 3.75 & 0 & 10.0 & 20.0 \\
28 & 12.4 & 0 & 12.1 & 13.75 & 10.0 & 7.2 & 0 \\
\hline & & & & & & &
\end{tabular}


591 Dataset S1. Continued

592

\begin{tabular}{cccccccc}
\hline Date & NP1 & NP2 & NP3 & NP4 & NP5 & NP6 & NP7 \\
\hline 29 & 19.6 & 18.0 & 21.3 & 0 & 0 & 8.2 & 15.0 \\
30 & 9.2 & 10.0 & 20.0 & 7.5 & 0 & 4.0 & 35.0 \\
31 & 13.4 & 22.0 & 19.1 & 0 & 0 & 1.3 & 35.0 \\
\hline
\end{tabular}

593

594 Note: NP1, NP2, NP3, NP4, NP5, NP6, and NP7 represent Doi Phu Kha, Mae Charim, Nanthaburi, 595 Tham Sa Koen, Sri Nan, Khun Sathan, and Doi Pha Klong national parks, respectively.

596 (http://www.parophrae.com/paro13kpi/index.php/45-2558)

597

598 Dataset S2. Rainfall data (mm.) in August 2018 from eight precipitation stations in Nan 599 province, Thailand

600

\begin{tabular}{|c|c|c|c|c|c|c|c|c|}
\hline Date & $\mathrm{S} 1$ & $\mathrm{~S} 2$ & S3 & S4 & S5 & S6 & S7 & S8 \\
\hline 1 & 1.9 & 1.0 & 0.1 & 1.3 & 0.0 & 0.0 & 0.0 & 0.0 \\
\hline 2 & 0.0 & 8.7 & 0.0 & 0.0 & 0.0 & 0.0 & 0.0 & 0.0 \\
\hline 3 & 0.0 & 59.0 & 3.2 & 22.6 & 81.0 & 0.0 & 0.0 & 6.4 \\
\hline 4 & 0.0 & 0.0 & 0.0 & 0.0 & 0.0 & 0.0 & 0.0 & 0.0 \\
\hline 5 & 0.0 & 0.7 & 0.0 & 0.0 & 5.9 & 0.0 & 0.5 & 0.0 \\
\hline 6 & 0.0 & 0.0 & 0.0 & 2.2 & 0.0 & 0.0 & 0.4 & 0.0 \\
\hline 7 & 0.0 & 0.0 & 5.4 & 0.0 & 0.0 & 0.0 & 1.2 & 0.0 \\
\hline 8 & 0.7 & 0.0 & 1.6 & 1.2 & 4.6 & 1.0 & 0.0 & 2.0 \\
\hline 9 & 9.3 & 7.1 & 19.3 & 26.3 & 8.6 & 5.2 & 22.5 & 7.0 \\
\hline 10 & 0.0 & 0.0 & 0.0 & 0.0 & 0.8 & 0.0 & 2.8 & 0.0 \\
\hline 11 & 0.0 & 0.0 & 4.2 & 0.0 & 0.7 & 0.0 & 1.2 & 0.0 \\
\hline 12 & 0.0 & 2.1 & 6.0 & 0.7 & 0.0 & 0.0 & 3.7 & 1.9 \\
\hline 13 & 0.8 & 10.6 & 9.8 & 6.4 & 12.5 & 0.0 & 8.3 & 8.4 \\
\hline 14 & 0.4 & 1.3 & 0.4 & 2.0 & 0.0 & 0.0 & 0.0 & 12.1 \\
\hline 15 & 0.0 & 0.0 & 0.0 & 0.0 & 0.0 & 0.0 & 0.0 & 2.7 \\
\hline 16 & 20.7 & 126.1 & 8.0 & 15.0 & 10.4 & 370.0 & 7.3 & 2.1 \\
\hline 17 & 69.2 & 72.0 & 106.1 & 111.0 & 151.5 & 65.0 & 90.2 & 153.0 \\
\hline 18 & 2.1 & 3.5 & 22.5 & 0.0 & 10.5 & 3.5 & 24.4 & 3.7 \\
\hline 19 & 0.0 & 0.0 & 0.5 & 0.0 & 0.0 & 0.0 & 0.0 & 0.0 \\
\hline 20 & 8.9 & 2.4 & 25.6 & 2.3 & 2.3 & 0.0 & 0.0 & 31.6 \\
\hline 21 & 8.8 & 4.4 & 4.8 & 6.7 & 5.0 & 0.0 & 2.3 & 2.0 \\
\hline 22 & 32.8 & 9.8 & 29.6 & 3.2 & 20.6 & 2.6 & 13.0 & 36.1 \\
\hline 23 & 1.8 & 2.6 & 9.1 & 1.4 & 0.0 & 9.0 & 2.8 & 0.0 \\
\hline 24 & 1.1 & 4.5 & 3.6 & 2.4 & 38.4 & 14.6 & 10.0 & 4.6 \\
\hline 25 & 8.7 & 0.0 & 1.3 & 2.0 & 8.7 & 3.5 & 0.0 & 1.6 \\
\hline 26 & 0.0 & 0.8 & 33.8 & 8.3 & 18.5 & 0.0 & 2.7 & 14.0 \\
\hline 27 & 6.9 & 0.1 & 5.4 & 22.4 & 31.0 & 2.2 & 7.2 & 1.3 \\
\hline 28 & 3.3 & 0.1 & 0.7 & 2.0 & 1.0 & 0.0 & 0.7 & 0.0 \\
\hline 29 & 2.5 & 4.8 & 6.6 & 6.0 & 3.9 & 3.7 & 12.4 & 4.7 \\
\hline 30 & 0.0 & 1.8 & 0.5 & 0.0 & 1.8 & 1.3 & 9.2 & 0.0 \\
\hline
\end{tabular}


601 Dataset S2. Continued

602

\begin{tabular}{ccccccccc}
\hline Date & S1 & S2 & S3 & S4 & S5 & S6 & S7 & S8 \\
\hline 31 & 0.0 & 0.3 & 3.9 & 0.0 & 6.2 & 0.0 & 20.2 & 3.3 \\
\hline
\end{tabular}

603

604 Note: S1, S2, S3, S4, S5, S6, S7, and S8 represent Muang, Thawangpha, Thung Chang, Pua, Song 605 Khwae, Santisuk, Chaloem Phra Kiat, and Chiang Klang precipitation stations, respectively.

606 (https://hydro-1.net/Data/HD-04/houly/rain_today_search.php?storage) 